Secretory TAT-peptide-mediated protein transduction of LIF receptor $\alpha$-chain distal cytoplasmic motifs into human myeloid HL-60 cells

Q. Sun, J. Xiong, J. Lu, S. Xu, Y. Li, X.P. Zhong, G.K. Gao and H.Q. Liu

The Brazilian Journal of Medical and Biological Research is partially financed by

\section{贵CNPq}

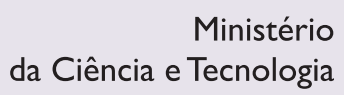

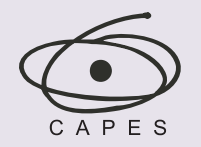

Ministério da Educação
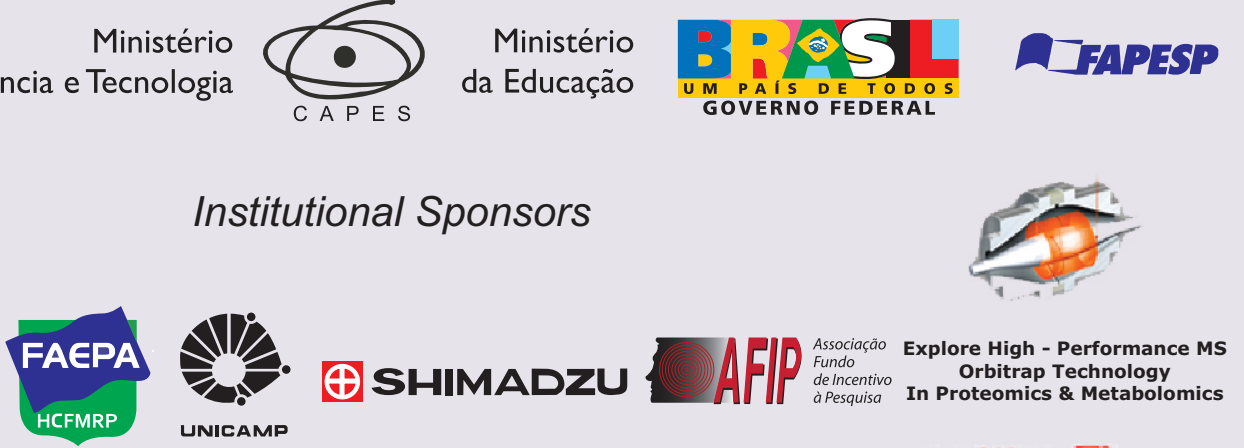

Institutional Sponsors

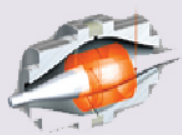

Ф SHIMADZU UNICAMP

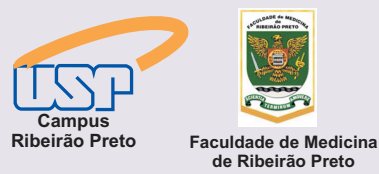

de Ribeirão Preto 


\title{
Secretory TAT-peptide-mediated protein transduction of LIF receptor $\alpha$-chain distal cytoplasmic motifs into human myeloid HL-60 cells
}

\author{
Q. Sun ${ }^{1,2 *}$, J. Xiong ${ }^{2 *}$, J. Lü ${ }^{3 *}$, S. Xu ${ }^{2}$, Y. Li ${ }^{4}$, X.P. Zhong ${ }^{1}$, \\ G.K. Gao ${ }^{1 *}$ and H.Q. Liu²* \\ ${ }^{1}$ Department of Hyperbaric Medicine, No. 401 Hospital of PLA, Qingdao, China \\ ${ }^{2}$ Department of Histology and Embryology, Faculty of Basic Medical Sciences, \\ Second Military Medical University, Shanghai, China \\ ${ }^{3}$ Office of Medical Education, Training Department, Second Military Medical University, Shanghai, China \\ ${ }^{4}$ State Food and Drug Administration of China, Huangdao Branch, Qingdao, China
}

\begin{abstract}
The distal cytoplasmic motifs of leukemia inhibitory factor receptor a-chain (LIFRa-CT3) can independently induce intracellular myeloid differentiation in acute myeloid leukemia (AML) cells by gene transfection; however, there are significant limitations in the potential clinical use of these motifs due to liposome-derived genetic modifications. To produce a potentially therapeutic LIFRa-CT3 with cell-permeable activity, we constructed a eukaryotic expression pcDNA3.0-TAT-CT3-cMyc plasmid with a signal peptide (ss) inserted into the N-terminal that codes for an ss-TAT-CT3-cMyc fusion protein. The stable transfection of Chinese hamster ovary $(\mathrm{CHO})$ cells via this vector and subsequent selection by Geneticin resulted in cell lines that express and secrete TAT-CT3-cMyc. The spent medium of pcDNA3.0-TAT-CT3-cMyc-transfected CHO cells could be purified using a cMycepitope-tag agarose affinity chromatography column and could be detected via SDS-PAGE, with antibodies against cMyc-tag. The direct administration of TAT-CT3-cMyc to HL-60 cell culture media caused the enrichment of CT3-cMyc in the cytoplasm and nucleus within 30 min and led to a significant reduction of viable cells $(P<0.05) 8 \mathrm{~h}$ after exposure. The advantages of using this mammalian expression system include the ease of generating TAT fusion proteins that are adequately transcripted and the potential for a sustained production of such proteins in vitro for future AML therapy.
\end{abstract}

Key words: Leukemia inhibitory factor; TAT-HIV1; Protein transduction domain; Acute myeloid leukemia; LIF receptor

\section{Introduction}

Leukemia inhibitory factor (LIF), as a member of the IL-6 family of cytokines, produces biological effects via binding to its receptor, which consists of a low-affinity LIF receptor $\alpha$-chain (referred to as LIFR $\alpha$ ) and a high-affinity subunit (referred to as gp130), both of which are shared with other IL-6 family cytokines (1). It has been reported that the 136-145-amino acid (aa) region of the distal Cterminal in cytoplasm, which contains 5-tyrosine residues (Y5) and several $Y X X Q$ motifs, is genetically conserved among gp130, LIFRa, and G-CSFR in numerous known cell lines (2,3). Thus, these motifs could initiate intracellular signaling by triggering LIFRa-gp130 heterodimerization or gp130-gp130/LIFR $\alpha$-LIFRa homodimerization via Janus kinase-signal transducer and activator of transcription (Jak/ STAT) and subsequently influence the growth or differentiation of leukemia cells (4). Intriguingly, because previous findings have confirmed that the enrichment of triple $Y X X Q$ motifs of the LIFRa cytoplasmic domain (LIFRa-CT3) via liposome transfection can inhibit proliferation and induce differentiation in human myeloid leukemia HL-60 cells $(5,6)$,

Correspondence: H.Q. Liu, Department of Histology and Embryology, Second Military Medical University, Shanghai, 200433, China. Fax: +86-21-8187-0958. E-mail: houqiliu@gmail.com and/or G.K. Gao, Department of Hyperbaric Medicine, No. 401 Hospital of PLA, Qingdao, 266071, China. Fax: +86-532-5187-0818. E-mail: anthonysun@cia.com

*These authors contributed equally to this study.

Received December 12, 2011. Accepted May 29, 2012. Available online June 22, 2012. Published September 3, 2012. 
we hypothesize that the LIFRa-CT3 polypeptide could also achieve a therapeutic effect intracellularly, thus repesenting a potential future peptide-targeting leukemia therapy.

Advances in protein delivery and the identification of several protein transduction domains have facilitated the delivery of proteins/peptides to cells or organs (7-10). The HIV-trans-activating transduction domain (TAT-PTD) has 11 aa (TAT-PTD 49-57: YGRKKRRQRRR) and can perform the intracellular delivery of proteins across the plasma membrane $(11,12)$. Although the mechanism of TAT-facilitated cellular uptake remains controversial and uncertain, TAT has been widely applied as a tool for protein transduction in vivo and in vitro after fusion with various full-length or truncated peptides (10-13). The technology of generating TAT fusion proteins requires the synthesis of a fusion protein in which TAT is linked to the molecule of interest via the use of a bacterial expression vector. In general, the TAT fusion protein is also linked to some sort of tag so as to facilitate its subsequent purification. The purified recombinant fusion protein could be directly added to mammalian cells in culture or injected in vivo into an animal (14). The above technique is generally highly applicable but laborious; in addition, a protein that is derived from a prokaryotic expression system is potentially more limited by its lack of splicing and the associated post-transcription processing systems or posttranslation modifying systems in comparison to eukaryotic expression systems.

In the present study, we have developed an alternative technology that offers advantages in terms of the application of TAT-mediated transduction techniques. We fused TAT-PTD $49-57$ with LIFRa-CT3 in the recombinant plasmid pcDNA3.0-ss-TAT-CT3-cMyc with a signal peptide (ss) inserted into the N-terminal. Next, the ss-TAT-CT3-cMyc fusion protein was expressed in Chinese hamster ovary $(\mathrm{CHO})$ cells before their culture supernatants were purified through an anti-cMyc agarose affinity column. When we compared the ss-TAT-CT3-cMyc fusion protein to its ssCT3-cMyc counterpart, the ss-TAT-CT3-cMyc fusion protein was found to be capable of being secreted from $\mathrm{CHO}$ cells and subsequently demonstrated a distinct capacity to be delivered into human myeloid leukemia HL-60 cells. Furthermore, we foresee that such transformed cells could be a sustained source of protein transduction domain (PTD) fusion peptides and other macromolecules in vivo.

\section{Material and Methods}

\section{Reagents}

Sall, Nhel, Xhol, and BamHI restriction enzymes were purchased from Invitrogen ${ }^{\mathrm{TM}}$ (USA). DNA polymerase, PCR purification kit, gel extraction kit, and plasmid miniprep kit were obtained from Dalian Takara ${ }^{\mathrm{TM}}$ (China). The pcDNA3.0 eukaryotic expression vector was purchased from Invitrogen. The rabbit anti-human LIFRa C-terminal antibody was obtained from Sigma ${ }^{\mathrm{TM}}$ (USA). All of the primers that were used in this study were synthesized by Sangon $^{\mathrm{TM}}$ (China).

\section{RT-PCR and vector construction}

Total RNA was extracted from $1 \times 10^{7} \mathrm{HL}-60$ cells (15). For pcDNA3.0-ss-CT3-cMyc, the ss-CT3-cMyc cDNA was amplified from the pcDNA3.0-gp190CT3 plasmid, which was kindly provided by Yang et al. (5), via a standard polymerase chain reaction with the forward primer $F 1$ (5'-CGCGGATCCGCCGCCACCATGGATTTTCAGGTG CAGATTTTCAGCTTCCTGCTAATCAGTGCCTCAGTC ATAATATCCAGAGGAGCTAGCCGCGTCGACTATC AGCCT-3') and reverse primer R (5'-CCGCTCGAGCTAC AGATCCTCTTCTGAGATGAGTTTTTGTTCATCGTTTGG TTTGTTC-3'). The forward primer F1 contained BamHI, Nhel, Sall (in bold above), and a 21-amino acid peptide, the sequence of which was originated from a human antibody heavy chain gene at the $\mathrm{N}$-terminals as a signal peptide (16) (as underlined above). The reverse primer $\mathrm{R}$ contained $\mathrm{Xhol}$ (in bold above) and a 10-aa cMyc epitope tag (17) (EQKLISEEDL) at the C-terminal for further protein purification (as underlined above). An approximately 471-bp amplified fragment was cut with $\mathrm{BamHI}$ and $\mathrm{Xhol}$ and later ligated into a pcDNA3.0 vector, which had been previously digested with $\mathrm{Bam} \mathrm{HI}$ and $\mathrm{Xhol}$, to create the corresponding pcDNA3.0-ss-CT3-cMyc expression vector.

For pcDNA3.0-ss-TAT-CT3-cMyc, two oligonucleotides were first synthesized and annealed so as to generate a double-stranded oligonucleotide with restriction sites for the Nhel and Sall restriction enzymes and to encode 11 aa (YGRKKRRQRRR) from the basic domain of HIV1TAT. The sequences were: 5'-CTAGCTATGGCAGGAAG AAGCGGAGACAGCGACGAAGAG-3' (the TAT sequence is underlined) and 5'-TCGACTCTTCGTCGCTGTCTCCGCTT CTTCGTGCCATAG-3'. We directly ligated the doublestranded nucleotide into the Nhel-Sall-digested pcDNA3.0ss-CT3-cMyc to create pcDNA3.0-ss-TAT-CT3-cMyc.

The proper orientations and sequences of pcDNA3.0ss-CT3-cMyc and pcDNA3.0-ss-TAT-CT3-cMyc were confirmed by automated sequencing.

\section{Transfection}

We transfected $\mathrm{CHO}$ cells using the FuGENE ${ }^{\circledR} 6$ Transfection Reagent (Roche Applied Science, USA) in a serumcontaining medium according to manufacturer guidelines. The transfection efficiencies using FuGENE Reagent and DNA ratios of $3: 1$ and $6: 1$ ( $\mu \mathrm{L}$ and $\mu \mathrm{g}$ for the amount of reagent and DNA, respectively) were similar and approximately twice as high as that obtained using a DNA ratio of 3:2. We carried out transfections with two plasmids: pcDNA3.0-ssCT3-cMyc and pcDNA3.0-ss-TAT-CT3-cMyc. We selected stable transformed cell lines, which were designated as CHO-CT3-cMyc and CHO-TAT-CT3-cMyc, using $2600 \mu \mathrm{g} / \mathrm{mL}$ Geneticin (Invitrogen) $24 \mathrm{~h}$ after transfection. The Geneticin sensitivities of the parent $\mathrm{CHO}$ cells were determined in pre- 
liminary experiments. The transformed cells were maintained in the same antibiotic concentrations.

\section{Cell culture}

$\mathrm{CHO}$ cells were maintained in RPMI-1640 medium (Gibco-BRL, Germany) containing 10\% fetal bovine serum (FBS, Gibco, Scotland), $100 \mathrm{U} / \mathrm{mL}$ penicillin, and $100 \mu \mathrm{g} /$ $\mathrm{mL}$ streptomycin (Gibco-BRL) at $37^{\circ} \mathrm{C}$ and $5 \% \mathrm{CO}_{2}$ in a humidified incubator (18).

The human myeloid leukemia HL-60 cell line was purchased from Cell Bank, Chinese Academy of Sciences (China), and cultured as described elsewhere (19).

\section{Protein expression and purification}

Stably transfected CHO-CT3-cMyc and CHO-TAT-CT3cMyc cells $\left(1.0 \times 10^{3}\right.$ cells $\left./ \mathrm{mL}\right)$ were cultured in $20 \mathrm{~mL}$ RPMI-1640 medium supplemented with $10 \%$ FBS, penicillin/streptomycin, and $2600 \mu \mathrm{g} / \mathrm{mL}$ Geneticin in a $75-\mathrm{cm}^{2}$ culture flask. When the cultures were near confluence, the medium was replaced with $15 \mathrm{~mL}$ serum-free RPMI-1640 without Geneticin. The medium was collected after $48-72 \mathrm{~h}$ of incubation at $37^{\circ} \mathrm{C}$ and centrifuged at approximately 2400 $g$ for $15 \mathrm{~min}$. The supernatant was used immediately or kept at $-20^{\circ} \mathrm{C}$. The process was repeated until $1 \mathrm{~L}$ supernatant was collected. After collection, the culture supernatants were combined and filtered through a $0.45-\mu \mathrm{m}$ filter so as to remove cells and cell debris. Next, the supernatants were loaded onto a gel filtration chromatography column that had been conjugated with anti-cMyc agarose according to manufacturer protocols (A7470, Sigma-Aldrich, Germany). The purified protein was eluted with $0.1 \mathrm{M}$ ammonium hydroxide at $\mathrm{pH} 11$ to 12 and subsequently neutralized with 1 $\mathrm{N}$ acetic acid. The concentration of the fusion protein was determined using a BCA protein assay kit (Pierce, USA) and bovine serum albumin as standard.

\section{Western blots}

For the Western blots, we cultured CHO-CT3-cMyc or CHO-TAT-CT3-cMyc cells in serum-free medium. We harvested the medium at near confluence, concentrated it using a 3000 MWCO Microcon Centrifugal Filter Device ${ }^{\mathrm{TM}}$ (Millipore, USA) at $4^{\circ} \mathrm{C}(20)$, separated it by $15 \%$ SDSPAGE, and electroblotted it onto polyvinylidene difluoride (PVDF) membranes (15). For immunoblotting, we used a 9E10 anti-Myc epitope tag monoclonal antibody (SC-40, Santa Cruz Biotechnology, USA) at a dilution of 1:2000 and a secondary peroxidase-labeled anti-mouse IgG antibody at a dilution of 1:5000. For the blocking and dilution of the antibodies, we used 1X TBS/Casein Blocker (Bio-Rad, USA). Protein molecular weight markers were purchased from Beyotime (P0062, China).

\section{Exposure of HL-60 cells to purified fusion proteins}

To test the transduction of the CT3-cMyc or TAT-CT3cMyc fusion protein, we first cultured $\mathrm{HL}-60$ cells as described in Ref. 19. Before the direct administration of purified fusion proteins, the HL-60 cells were centrifuged and rinsed three times with PBS to eliminate any possible FBS-induced effects. Next, the cells were re-seeded on six-well plates at a concentration of $1 \times 10^{5}$ well with serum-free medium and received fusion proteins at different final concentrations. The same volume of PBS was also added as an internal control. In general, we fixed the corresponding $\mathrm{HL}-60$ cultures after $30 \mathrm{~min}, 4 \mathrm{~h}$, and $8 \mathrm{~h}$ of exposure and examined them under a fluorescence microscope.

\section{Fluorescence microscopy}

The pre-staining treatment of non-adherent HL-60 cells $\left(\sim 1 \times 10^{5} / \mathrm{mL}\right)$ was carried out as described in Ref. 21 . The smears were incubated with a mouse-anti-human cMyc primary antibody that had been diluted in blocking serum (1:50), incubated for $30 \mathrm{~min}$ at $37^{\circ} \mathrm{C}$, and then rinsed for 5 min in $4 \mathrm{X}$ saline-sodium citrate/ $0.1 \%$ Tween 20 . This process was repeated two additional times. The blocking step was repeated before the smears were incubated with a fluorescein isothiocyanate-conjugated secondary antibody that had been diluted in blocking solution (1:200) and subsequently rinsed as above. The smears were eventually mounted in an anti-fade solution for analysis. The nuclei of HL-60 cells were counterstained with DAPI, visualized with a fluorescence microscope (Leica, Germany) at 630X magnification, and photographed using a charge-coupled camera.

\section{Cell viability and statistical analysis}

The viability of HL-60 cells was assessed with the cell counting kit-8 (CCK-8; Beyotime) assay as described by Ting et al. (22) and Lang et al. (23). The HL-60 cells were centrifuged and rinsed three times with PBS so as to eliminate any possible FBS-induced effect. Next, the cells were re-seeded on a 96-well plate at a concentration of $1 \times 10^{4}$ cells per well and either exposed to the CT3-cMyc protein or to the TAT-CT3-cMyc protein $(30 \mu \mathrm{M})$ for $8 \mathrm{~h}$ with serumfree medium. The same volume of PBS was also added as an internal control. The culture medium was then changed to RMPI 1640 with $10 \%$ FBS. The CCK-8 reagents were added at $24,48,72$, and $96 \mathrm{~h}$, respectively, after adding the CT3-cMyc protein or the TAT-CT3-cMyc protein. Plates were incubated at $37^{\circ} \mathrm{C}$ for $1 \mathrm{~h}$ and absorbance at $450 \mathrm{~nm}$ was recorded.

The cell viability tests were performed at least three times, and the data are reported as means \pm SEM. The results were considered to be statistically significant if $P$ $<0.05$ as determined by the chi-square test. The analysis was conducted using SPSS 17.0.

\section{Results}

Transfection and establishment of cell lines that express and secrete TAT-CT3-cMyc and CT3-cMyc

The full-length LIFRa-CT3 consists of 118 aa residues 
(5). To produce a cell-permeable LIFRa-CT3 with an epitope tag (-cMyc), a eukaryotic expression vector bearing the TAT-CT3-cMyc gene was constructed. A recombinant plasmid without a TAT domain was also constructed as the control (Figure 1). Both pcDNA3.0-ss-TAT-CT3-cMyc and pcDNA3.0-ss-CT3-cMyc plasmids are designed for high-level stable expression in mammalian hosts and for the secretion of proteins that are fused at the $\mathrm{N}$-terminal to the human antibody heavy chain gene.

We established stably transfected cell lines that expressed the two fusion proteins (the $\sim 18.6-\mathrm{kDa}$ ss-TATCT3-cMyc and 17.0-kDa ss-CT3-cMyc). CHO-CT3-cMyc and $\mathrm{CHO}-\mathrm{TAT}-\mathrm{CT} 3-\mathrm{cMyc}$ cells were observed to secrete CT3-cMyc and TAT-CT3-cMyc, respectively, into the culture medium. Western blots of the concentrated, serum-free spent medium of the CHO-CT3-cMyc and CHO-TAT-CT3cMyc cells, which were prepared using a monoclonal antibody to cMyc, revealed a single band of protein with the same migration as that observed in SDS-PAGE. The serum-free spent medium of wild-type $\mathrm{CHO}$ cells was also concentrated and labeled by the same procedure as an internal control. The molecular weight of CT3-cMyc or TAT-cMyc-CT3-cMyc was approximately $18 \mathrm{kDa}$, whose specificity was confirmed to one protein band extracted from the spent medium of wild-type $\mathrm{CHO}$ cells as the negative control (Figure 2).

\section{TAT-mediated delivery of CT3-cMyc protein to human myeloid HL-60 cells}

To evaluate the ability of TAT-related CT3 delivery to human myeloid leukemia HL-60 cells, we compared the transmembrane delivery of CT3-cMyc and TAT-CT3-cMyc after their direct administration to the serum-free medium of the HL-60 cells at various concentrations or times. Purified CT3-cMyc and TAT-CT3-cMyc fusion proteins were added to a total of $1 \times 10^{5} \mathrm{HL}-60$ cells each so as to achieve final concentrations of 10,30 , and 50 $\mu \mathrm{g} / \mathrm{mL}$ at $30 \mathrm{~min}, 4 \mathrm{~h}$, and $8 \mathrm{~h}$. The identical PBS volumes were added to HL-60 cells to act as controls. The intracellular distributions of these fusion proteins were examined by immunofluorescence.

On a time basis, when the administered doses of both fusion proteins were $30 \mu \mathrm{g} /$ $\mathrm{mL}$, which were similar to the PBS controls (Figure 3), no cMyc-positive cells were found in the HL-60 cells that were obtained from the CT3-cMyc-treated group after $4 \mathrm{~h}$ (Figure 3 ); however, cMyc-positive cells were readily detected in the HL-60 cells that were obtained from the TAT-CT3-cMyc-treated group $30 \mathrm{~min}$ after their administration (Figure 3). TAT-CT3-
cMyc proteins were primarily found in the nuclei of HL-60 cells. The TAT-CT3-cMyc protein levels, which were calculated based on the number and fluorescence intensity of cMyc-positive cells, peaked at around $30 \mathrm{~min}$ to $1 \mathrm{~h}$ after their administration and gradually decreased thereafter, although they were still detectable $8 \mathrm{~h}$ after administration. These data indicate that TAT-CT3-cMyc but not CT3-cMyc possesses the ability to deliver LIFRa-CT3.

On a dose-dependent basis, because we knew the approximate timing of the transmembrane delivery of TAT-CT3-cMyc, we further explored the dose-response relationships between the fusion protein dosages and fluorescence intensities of cMyc-positive cells after $1 \mathrm{~h}$ of TAT-CT3-cMyc fusion protein administration. cMyc-positive cells were readily detected in the HL-60 cells obtained from the TAT-CT3-cMyc-treated group in the $10-\mu \mathrm{g} / \mathrm{mL}$ sample (Figure 4). TAT-CT3-cMyc proteins were primarily found in the nuclei of HL-60 cells. As the protein concentrations were increased, the TAT-CT3-cMyc protein concentrations, which were calculated on the basis of the fluorescence intensity

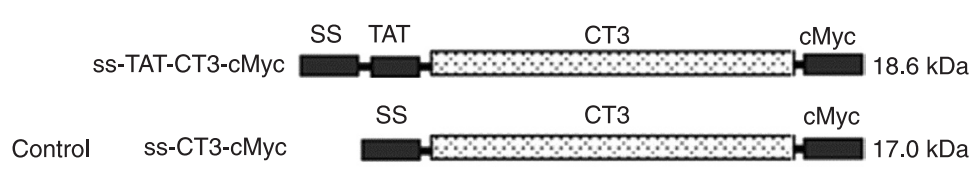

Figure 1. sS-TAT-CT3-cMyc and control ss-CT3-cMyc fusion proteins.

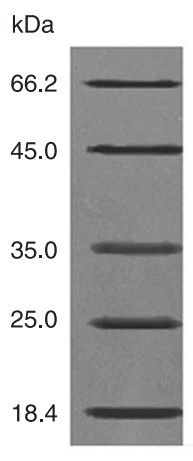

1

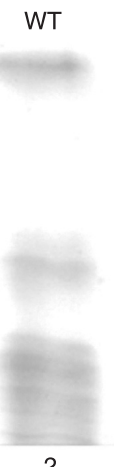

2

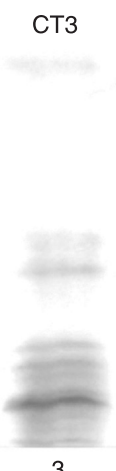

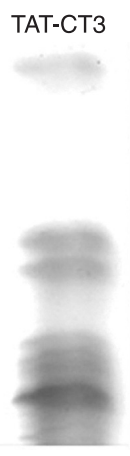

4

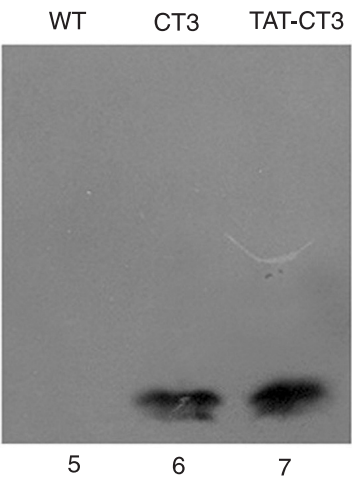

Figure 2. SDS-PAGE and Western blot of the concentrated spent media of CHO-CT3-cMyc and CHO-TAT-CT3-cMyc cells. The same volume of wild-type (WT) $\mathrm{CHO}$ cell spent medium was added as a blank control. The migration of both proteins from the spent medium were similar and indicated an apparent molecular mass of approximately $18 \mathrm{kDa}$. Both transformed cells lines were maintained in RPMI-1640 medium supplemented with $10 \%$ FBS to near confluence in $75-\mathrm{cm}^{2}$ culture flasks. The medium was replaced with $15 \mathrm{~mL}$ serumfree RPMI-1640, which was collected $72 \mathrm{~h}$ later, centrifuged, and concentrated. SDS-PAGE and immunoblots were prepared as described in the Material and Methods section. Lanes 1-4 = Coomassie blue staining; lanes 5-7 = immunoblots using a cMyc-epitope-tag specific antibody; lane $1=$ molecular weight markers; lanes 2-7 = $30 \mu \mathrm{L}$ of concentrated spent media. 
$30 \mathrm{~min}$
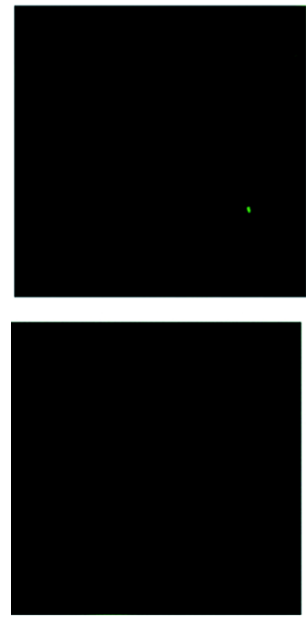

CT3-cMyc

TAT-СT3-сMyc

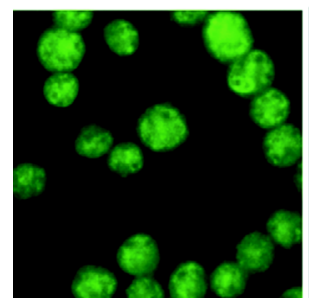

$4 \mathrm{~h}$
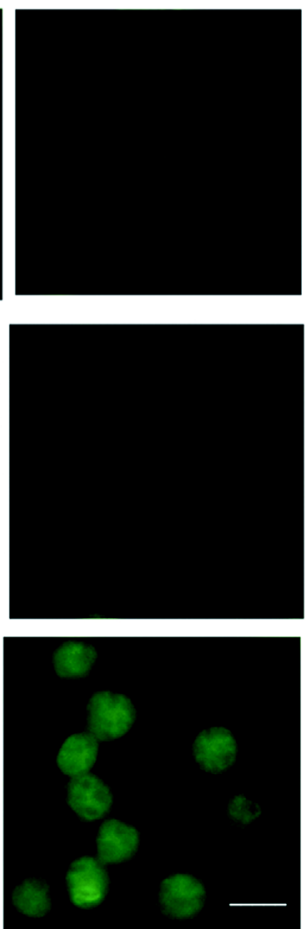

$8 \mathrm{~h}$
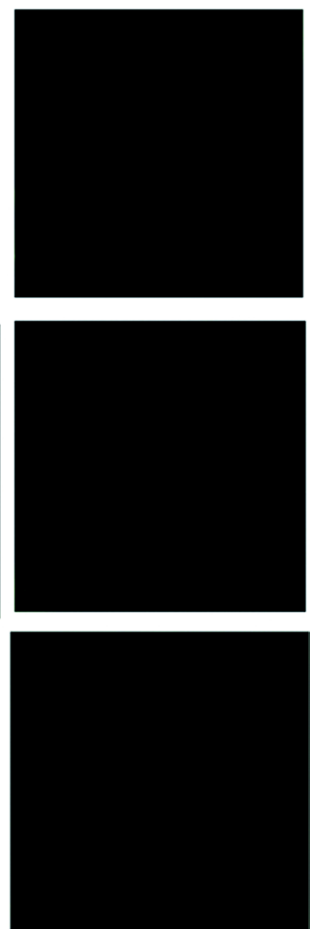

Figure 3. TAT-CT3-cMyc fusion proteins demonstrate transmembrane delivery into HL-60 cells after different times. HL-60 cells were fixed $30 \mathrm{~min}, 4 \mathrm{~h}$, and $8 \mathrm{~h}$ after PBS, CT3-cMyc, or TAT-CT3-cMyc was added directly to the medium. The intracellular distribution of the fusion protein or control was examined by immunofluorescence using an anti-cMyc antibody. One hundred cells were analyzed at each time, and the representative images are shown. Scale bar $=15 \mu \mathrm{m}$.

$10 \mu \mathrm{g} / \mathrm{mL}$

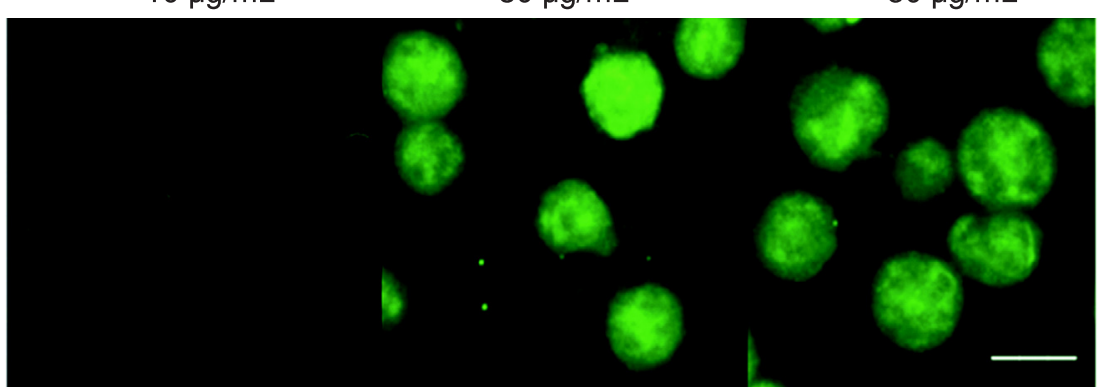

Figure 4. Ideal dosage for transmembrane delivery of TAT-CT3-cMyc fusion protein into $\mathrm{HL}-60$ cells. The human myeloid HL-60 cells were cultured with serum-free medium just before receiving a final concentration of 10,30 , and $50 \mu \mathrm{g} / \mathrm{mL}$ TAT-CT3-cMyc fusion protein. The intracellular distribution of the fusion proteins was examined by immunofluorescence using an anti-cMyc antibody $1 \mathrm{~h}$ after administration. One hundred cells were analyzed at each time, and representative images are shown. Scale bar $=15 \mu \mathrm{m}$. of cMyc-positive cells, peaked at a concentration of $30 \mu \mathrm{g} /$ $\mathrm{mL}$ and did not significantly change when compared to the increased concentration up to $50 \mu \mathrm{g} / \mathrm{mL}$. These data indicate that approximate $30 \mu \mathrm{g} / \mathrm{mL}$ TAT-CT3-cMyc fusion protein was the ideal and saturated dosage close enough in this experimental design for the transmembrane delivery of $1 \times 10^{5} \mathrm{HL}-60$ cells in vitro.

TAT-CT3-cMyc-induced change in HL-60 cell viability To assess the effect of delivered LIFRa-CT3 fusion 
protein in HL-60 cells, we compared the cell viability changes after the direct administration of fusion proteins to the serum-free medium of the HL-60 cells at $30 \mu \mathrm{g} / \mathrm{mL}$ for $8 \mathrm{~h}$. No significant difference in cell viability was observed after $24 \mathrm{~h}$ of incubation with PBS, CT3-cMyc protein or TAT-CT3-cMyc protein ( $P>0.05)$. A significant decrease in cell viability was observed for HL-60 cells incubated with TAT-CT3-cMyc for 48, 72 , and $96 \mathrm{~h}$ compared to cells incubated with PBS or CT3-cMyc protein ( $\mathrm{P}<0.05)$, based on the CCK-8 assay (Figure 5). A significant decrease in cell viability was not observed in the presence of CT3-cMyc or of an equal amount of PBS as the internal control of wild-type HL-60 cells.

\section{Discussion}

In the present study, we have shown that an LIFRaCT3 fusion protein that bears the protein transduction domain of the HIV1-TAT protein, a signal peptide from the human antibody heavy chain gene, and a cMycepitope-tag can be derived from stably transformed eukaryotic expression $\mathrm{CHO}$ cells and subsequently delivered into human myeloid leukemia HL-60 cells.

LIF is so named because it can induce a differentiation of the M1 murine myeloid leukemia cell line $(24,25)$. LIFRa-CT3, as reported in our previous research, was observed to have the capacity to activate signal transducer and activator of transcription3 (STAT3) in HL60 cells by transfection, to initiate LIF-related intracellular signaling, and to facilitate both increasing differentiation and decreasing proliferation $(5,26-28)$; however, transfection or virus-mediated gene delivery may cause an irreversible genetic modification, which makes these deliveries significantly unacceptable for possible clinical practice $(29,30)$. Peptide-based cell delivery systems are greatly expanded by the recognition of PTDs and synthetic peptides with translocation properties $(14,31,32)$. In the present study, we have shown that the TAT-CT3-cMyc fusion protein can be expressed in eukaryotic system $\mathrm{CHO}$ cells, secreted into the medium, and efficiently purified. The purified protein was shown to be able to penetrate not only cells but also the nuclear membranes of HL-60 cells (33).

The technique for the production of TAT-related fusion proteins in general requires the synthesis and purification of such proteins using prokaryotic expression systems (34-36). As an alternative, we have developed a method based on the application of a mammalian expression vector, pcDNA3.0. This vector is designed for high-level expression and can be inserted into other domains so as to regenerate novel properties. In our particular case, the resultant novel property was the ability of the fusion proteins to be secreted out of the $\mathrm{CHO}$ cells into serum-free medium supernatants, to be effectively concentrated and directly administered to target leukemia cell. As a prototype, we developed stable

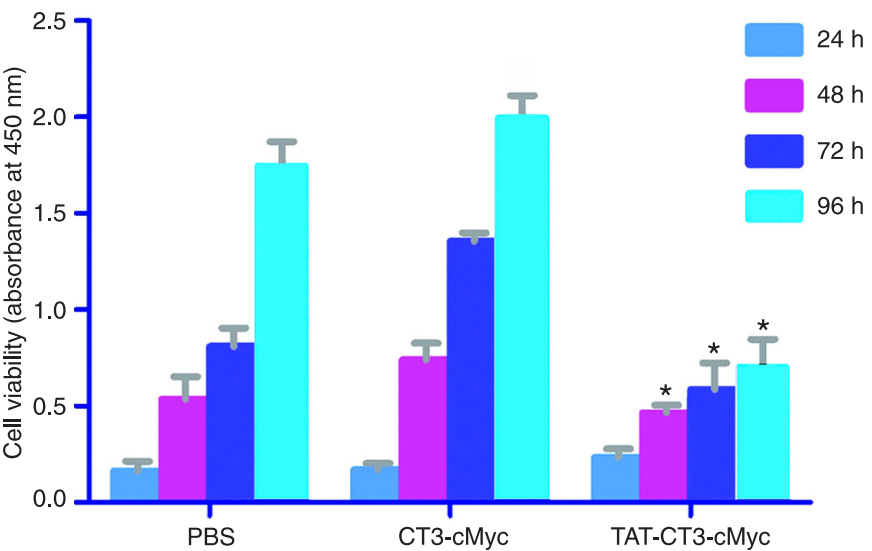

Figure 5. Viability of HL-60 cells measured by the cell counting kit-8 (CCK-8) assay. PBS only, CT3-cMyc or TAT-CT3-cMyc fusion protein was added to serum-free media of HL-60 cells for $8 \mathrm{~h}$ before the media were replaced with normal media (RMPI 1640 with $10 \%$ FBS). Cell viability was measured using the CCK-8 assay at $24,48,72$, and $96 \mathrm{~h}$ after adding the CT3-cMyc or TAT-CT3-cMyc protein ( $\mathrm{N}=3$ for each group). A significant decrease in cell viability was observed for $\mathrm{HL}-60$ cells incubated with TAT-CT3-cMyc for $48 \mathrm{~h}, 72 \mathrm{~h}$ and $96 \mathrm{~h}$ compared to cells incubated with PBS or CT3-cMyc protein at the same times. ${ }^{*} \mathrm{P}$ $<0.05$; chi-square test.

transformed cell lines (37) with easily detected sensitivities in fixed cells to illustrate the advantages of this technique.

The purity of the recombinant TAT-CT3-cMyc was greater than $90 \%$, as determined by SDS-PAGE. Previous studies have shown that secreted TAT fusion proteins are able to transduce target cells but with very low efficiency (37). According to our study, this is not always the case. The original structure of ss-TAT-CT3-cMyc without mutations was observed to be qualified enough to ensure the secretion of TAT-CT3-cMyc, which is vital in exploring the structure on which TAT-PTD is based. More specifically, the $30 \mu \mathrm{g} / \mathrm{mL}$ TAT-CT3-cMyc fusion protein concentration used in our study was highly effective in transmembrane location within an hour and was primarily located in the nucleus. We speculate that this phenomenon may have been due first to the location of TAT-PTD, which was located downstream of the signal peptide on the N-terminal, which, in turn, may have somehow transformed itself and may have protected the specific sites from being cleaved by endogenous furins $(38,39)$; second, the signal peptide of our study was never applied to the TAT-based eukaryotic expressing system, which may also contribute to the change in protein conformation leading to potential damage.

There are remaining unsolved problems in $\mathrm{CHO}$ cellbased TAT-fusion-protein production. The level of protein expression in $\mathrm{CHO}$-transfected cells constantly varies from cell to cell. Some researchers attribute this variation to the inherently stochastic nature of gene expression. The stochastic mechanism in gene expression operates in both prokaryotes and eukaryotes and may explain phenotypic 
variations in isogenic cell populations (40). In our observation, TAT-CT3-cMyc and CT3-cMyc were poorly expressed in the transformed $\mathrm{CHO}$ cells when determined by immunofluorescence using antibodies against cMyc-epitope-tag but highly detectable in a spent medium of corresponding $\mathrm{CHO}$ cells (data not shown). Hence, we further speculate that the gene involved in this liposome-based methodology may induce epigenetic alterations of host cells that make it hard to monitor the expression levels in $\mathrm{CHO}$ cells but may somehow not affect the efficiency when fusion proteins are capable of being secreted outside the host cells. Such evidence and reasoning make $\mathrm{CHO}$ cells, which are a eukaryotic expression system, an ideal tool for LIFRaCT3-based acute myeloid leukemia therapy.

The use of a mammalian secretory system to generate TAT-related fusion proteins facilitates their preparation because of its apparent soluble form and more reliable

\section{References}

1. Guo SY, Shen X, Yang J, Yuan J, Yang RL, Mao K, et al. TIMP-1 mediates the inhibitory effect of interleukin- 6 on the proliferation of a hepatocarcinoma cell line in a STAT3-dependent manner. Braz J Med Biol Res 2007; 40: 621-631.

2. Liu H, Dan J, Tang S, Wu S. Involving of the cytoplasmic region of leukemia inhibitory factor receptor alpha subunit, IL-6 related signal transducer-gp130 or fas death domain for MAPK p42/44 activation in HL-60 cell with LIF or anti-Fas IgG. Mol Cell Biochem 2001; 217: 113-120.

3. White UA, Stephens JM. Neuropoietin activates STAT3 independent of LIFR activation in adipocytes. Biochem Biophys Res Commun 2010; 395: 48-50.

4. Cardoso BA, Girio A, Henriques C, Martins LR, Santos C, Silva A, et al. Aberrant signaling in T-cell acute lymphoblastic leukemia: biological and therapeutic implications. Braz $J$ Med Biol Res 2008; 41: 344-350.

5. Yang L, Liu SR, Tang SP, Wang FM, Liu HQ. [Effects of the box-3 region of the LIFRalpha-chain cytoplasmic domain (gp190CT3) on the proliferation and differentiation of HL-60 cells]. Zhonghua Xue Ye Xue Za Zhi 2004; 25: 679-682.

6. Tomida M, Heike T, Yokota T. Cytoplasmic domains of the leukemia inhibitory factor receptor required for STAT3 activation, differentiation, and growth arrest of myeloid leukemic cells. Blood 1999; 93: 1934-1941.

7. Abes R, Arzumanov A, Moulton H, Abes S, Ivanova G, Gait $\mathrm{MJ}$, et al. Arginine-rich cell penetrating peptides: design, structure-activity, and applications to alter pre-mRNA splicing by steric-block oligonucleotides. J Pept Sci 2008; 14: 455-460.

8. Torchilin VP. Cell penetrating peptide-modified pharmaceutical nanocarriers for intracellular drug and gene delivery. Biopolymers 2008; 90: 604-610.

9. Johnson LN, Cashman SM, Kumar-Singh R. Cell-penetrating peptide for enhanced delivery of nucleic acids and drugs to ocular tissues including retina and cornea. Mol Ther 2008; 16: 107-114.

10. Pan C, Lu B, Chen H, Bishop CE. Reprogramming human fibroblasts using HIV-1 TAT recombinant proteins OCT4, transcriptional structure so that they can be directly added to cultured cells. The positive use of LIF in vitro, which is defined as promoting myeloid differentiation and inhibiting cell proliferation, can be achieved by TAT-CT3-based extracellular administration of TAT-CT3-cMyc to HL-60 cells. We have also presumed that the therapeutic application of this technique, whereby parent cells have been transfected with constructs that code for the PTD fusion proteins, could benefit leukemia patients (14).

\section{Acknowledgments}

Research supported by grants from the Science and Technology Commission of Shanghai Municipality (No. 09431901100) and Key Program of High and Novel-Tech Building in Clinical Medicine for the People's Liberation Army (\#2010GXJS023).

SOX2, KLF4 and c-MYC. Mol Biol Rep 2010; 37: 21172124.

11. Jiang $L$, Ma $Y$, Wang J, Tao $X$, Wei D. The transduction of His-TAT-p53 fusion protein into the human osteogenic sarcoma cell line (Saos-2) and its influence on cell cycle arrest and apoptosis. Mol Biol Rep 2008; 35: 1-8.

12. Hou Y, Zou J. Delivery of HSF1(+) protein using HIV-1 TAT protein transduction domain. Mol Biol Rep 2009; 36: 22712277.

13. Dietz GP, Bahr M. Delivery of bioactive molecules into the cell: the Trojan horse approach. Mol Cell Neurosci 2004; 27 : 85-131.

14. Barka T, Gresik ES, Henderson SC. Production of cell lines secreting TAT fusion proteins. J Histochem Cytochem 2004; 52: 469-477.

15. Fang ZH, Dong CL, Chen Z, Zhou B, Liu N, Lan HF, et al. Transcriptional regulation of survivin by c-Myc in BCR/ABLtransformed cells: implications in anti-leukaemic strategy. $J$ Cell Mol Med 2009; 13: 2039-2052.

16. Li B, Wang H, Dai J, Ji J, Qian W, Zhang D, et al. Construction and characterization of a humanized anti-human CD3 monoclonal antibody $12 \mathrm{~F} 6$ with effective immunoregulation functions. Immunology 2005; 116: 487-498.

17. Ren $X Q$, Furukawa $T$, Aoki $S$, Nakajima $T$, Sumizawa $T$, Haraguchi $M$, et al. Glutathione-dependent binding of a photoaffinity analog of agosterol A to the C-terminal half of human multidrug resistance protein. J Biol Chem 2001; 276: 23197-23206.

18. Merjan AJ, Kanashiro CA, Krieger JE, Han SW, Paiva AC. Ligand-induced endocytosis and nuclear localization of angiotensin II receptors expressed in $\mathrm{CHO}$ cells. Braz J Med Biol Res 2001; 34: 1175-1183.

19. Pompeia C, Cury-Boaventura MF, Curi R. Arachidonic acid triggers an oxidative burst in leukocytes. Braz J Med Biol Res 2003; 36: 1549-1560.

20. Dostal L, Chen CY, Wang AH, Welfle H. Partial B-to-A DNA transition upon minor groove binding of protein Sac7d monitored by Raman spectroscopy. Biochemistry 2004; 43: 
9600-9609.

21. Bach JP, Borta $H$, Ackermann W, Faust F, Borchers $O$, Schrader $\mathrm{M}$. The secretory granule protein syncollin localizes to HL-60 cells and neutrophils. J Histochem Cytochem 2006; 54: 877-888.

22. Ting L, Bo W, Li R, Chen X, Wang Y, Jun Z, et al. AMPactivated protein kinase supports the NGF-induced viability of human HeLa cells to glucose starvation. Mol Biol Rep 2010; 37: 2593-2598.

23. Lang $\mathrm{Q}$, Zhang $\mathrm{H}$, Li J, Xie F, Zhang $\mathrm{Y}$, Wan B, et al. 3-Hydroxyflavone inhibits endogenous Aurora $B$ and induces growth inhibition of cancer cell line. Mol Biol Rep 2010; 37: 1577-1583.

24. Tomida M, Yamamoto-Yamaguchi Y, Hozumi M. Purification of a factor inducing differentiation of mouse myeloid leukemic $\mathrm{M} 1$ cells from conditioned medium of mouse fibroblast L929 cells. J Biol Chem 1984; 259: 10978-10982.

25. Gearing DP, Gough NM, King JA, Hilton DJ, Nicola NA, Simpson RJ, et al. Molecular cloning and expression of cDNA encoding a murine myeloid leukaemia inhibitory factor (LIF). EMBO J 1987; 6: 3995-4002.

26. Liu H, Liu S, Tang S, Ji K, Wang F, Hu S. Molecular analysis of signaling events mediated by the cytoplasmic domain of leukemia inhibitory factor receptor alpha subunit. Mol Cell Biochem 2004; 258: 15-23.

27. Liu H, Tang S, Liu S. [Signal of the cytoplasmic regions of leukemia inhibitory factor receptor (LIFR) alpha-subunit and gp130 involves Stat3 activation in leukemic U937 cells]. Zhonghua Xue Ye Xue Za Zhi 1999; 20: 621-623.

28. Sun Q, Wang J, Xiong J, Yang L, Liu H. Free LIF receptor alpha-chain distal cytoplasmic motifs enhance Jak2-independent STAT3 phosphorylation and induce differentiation in HL-60 cells. Oncol Rep 2011; 26: 399-404.

29. Schwartz JJ, Zhang S. Peptide-mediated cellular delivery. Curr Opin Mol Ther 2000; 2: 162-167.

30. Ford KG, Souberbielle BE, Darling D, Farzaneh F. Protein transduction: an alternative to genetic intervention? Gene Ther 2001; 8: 1-4.

31. Denicourt C, Dowdy SF. Protein transduction technology offers novel therapeutic approach for brain ischemia. Trends Pharmacol Sci 2003; 24: 216-218.

32. Zhao J, Gao P, Xiao W, Fan LQ, Wang FJ, Li SX, et al. A novel human derived cell-penetrating peptide in drug delivery. Mol Biol Rep 2011; 38: 2649-2656.

33. Gump JM, June RK, Dowdy SF. Revised role of glycosaminoglycans in TAT protein transduction domain-mediated cellular transduction. J Biol Chem 2010; 285: 1500-1507.

34. Wu Y, Ren C, Gao Y, Hou B, Chen T, Zhang C. A novel method for promoting heterologous protein expression in Escherichia coli by fusion with the HIV-1 TAT core domain. Amino Acids 2010; 39: 811-820.

35. Wang $\mathrm{Y}$, Lin H, Lin S, Qu J, Xiao J, Huang $\mathrm{Y}$, et al. Cellpenetrating peptide TAT-mediated delivery of acidic FGF to retina and protection against ischemia-reperfusion injury in rats. J Cell Mol Med 2010; 14: 1998-2005.

36. Muthumani K, Lambert VM, Shanmugam M, Thieu KP, Choo AY, Chung JC, et al. Anti-tumor activity mediated by protein and peptide transduction of HIV viral protein R (Vpr). Cancer Biol Ther 2009; 8: 180-187.

37. Flinterman M, Farzaneh F, Habib N, Malik F, Gaken J, Tavassoli M. Delivery of therapeutic proteins as secretable TAT fusion products. Mol Ther 2009; 17: 334-342.

38. Gaken J, Jiang J, Daniel K, van Berkel E, Hughes C, Kuiper $\mathrm{M}$, et al. Fusagene vectors: a novel strategy for the expression of multiple genes from a single cistron. Gene Ther 2000; 7: 1979-1985.

39. Tikhonov I, Ruckwardt TJ, Berg S, Hatfield GS, David PC. Furin cleavage of the HIV-1 Tat protein. FEBS Lett 2004; 565: 89-92.

40. McAdams $\mathrm{HH}$, Arkin A. Stochastic mechanisms in gene expression. Proc Natl Acad Sci U S A 1997; 94: 814-819. 